\title{
Effects of Electronics on Human Health
}

ISSN: 2637-8078

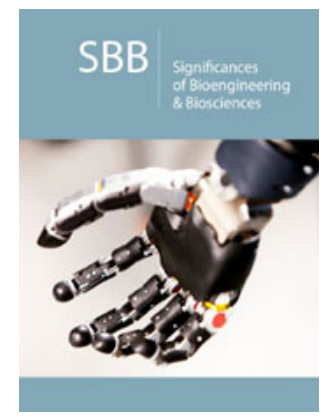

*Corresponding author: Arun Singh, Department of Community Medicine, Rohilkhand Medical College \& Hospital, India

Submission: 侮July 12, 2019

Published: 罡August 6,2019

Volume 3 - Issue 4

How to cite this article: Arun Singh Masood S A. Effects of Electronics on Human Health. Significances Bioeng Biosci.3(4). SBB.000568.2019.

DOI: 10.31031/SBB.2019.03.000568

Copyright@ Arun Singh, This article is distributed under the terms of the Creative Commons Attribution 4.0 International License, which permits unrestricted use and redistribution provided that the original author and source are credited.

\author{
Arun Singh* and Masood Siddiqui A \\ Department of Community Medicine, India
}

Abbreviations: LEDs: Light Emitting Diodes; PCB: Printed Circuit Board; ASIC: Application-Specific Integrated Circuit; DSP: Digital Signal Processor; FPGA: Field-Programmable Gate Array

\section{Mini Review}

Electronic devices, machines, home appliances, gadgets, accessories, related components, integrated parts, electronic systems create electromagnetic fields in the surroundings [1]. In these electronics, the electrons have very important and fundamental roles. Digital electronics, analogue electronics, microelectronics, circuit design, integrated circuits, optoelectronics, semiconductors, embedded systems generate the electromagnetic waves. The electrical circuits involve active and passive components like resistors, transistors, capacitors, microcontrollers, inductors, transformers, relays, circuit breakers, switches, motors, batteries, fuses, diodes, light-emitting diodes (LEDs), vacuum tubes, integrated circuits, printed circuit board (PCB), amplifiers, radio receiver, oscillators, logic gates, adders, flip-flops, counters, multiplexers, Schmitt triggers, microprocessors, microcontrollers, application-specific integrated circuit (ASIC), digital signal processor (DSP), field-programmable gate array (FPGA) and many other associated passive electrical components including the interconnection technologies and these also generates electromagnetic fields [2]

Following devices and machines are available in the markets and have been used for various purposes in day today's life by the humans [3]. The above components are integrated into the below-mentioned machines, devices and the electric currents flow and create the electromagnetic waves in the surrounding and affect the health status of human beings working with the help of these machines. The machines, gadgets and devices like Television, Laptops/Computers, Cameras, Nintendo, Kindle Fire, PSP, Oven, Smart Phones, Apple devices, Hair Straightener, Electric Lamp, Washing Machine, Bolometer, Music systems/ devices, Fans, Cell Phones, Play Station/Gaming consoles, Printer, Tablets/gadgets, Pen Drives, Kindle devices, Projectors, Google Glass, Blu Ray/CD/DVD Players, Inverter, Radio, Electronic Watches, Phone Charger, Camera and Recorders, The Remote Control, Stereo Equalizer, Memory Card, Telephones, Refrigerator, Sega Dreamcast/ cast devices, Speakers, Odometer, Recordable CD Player, Cassette Deck, Hair Dryer, Electric Shaver, Wii U, Adapter/ Chargers, ATM Machine, Blender, Stereo receiver, Flow Guard Water Level Controller, Virtual Reality Devices, Headphones, Baby Monitors, GPS Navigation Devices, PSP Go, Air Conditioner, Microphone, Scanner, Turntables, iQue Player, Amazon Echo, Robots, Artificial Intelligence, imaging machines, health care devices, diagnostic devices and many more [4] can emit radiations can compel humans to adopt certain constant postures for long duration or can create certain circumstances prone for accidents or physical or chemical injuries. All these devices are useful but have associated health hazards so only judicial use of these can prevent associated disorders. There are possible risks and hazards to human health due to overuse and inappropriate use of electronic devices, gadgets electronic home appliances and machines and the judicial use will be helpful in prevention and control of these risks and hazards. Following are the important aspects of life affected by the use of electronics. Excessive Brightness from screens of the gadgets can cause dizziness and light-headedness, tiredness, headaches, insomnia, vertigo, sleep deprivation [5]. Sitting and resting posture while using gadgets can cause back pains, neck pains, shoulder pain, tendon pains, nerve injuries are also common among the person working for a long duration without changing their postures. Accidents are possible while using gadgets and headphones/earbuds e.g. using the earphones 
for a long duration can cause permanent or temporary hearing loss and make the person venerable to the accidents. There are examples of accidents while crossing railway tracks and roads while crossing the roads and using earphones [6]. Besides the exposure of cosmic rays coming from the sky the radiations from towers of telecommunication networking, internet services also affecting the health of human beings and the cumulative effects are responsible for radiation-related following health disorders.

Radiation causes the burns, heat exhaustion, heatstroke, heat cramps, dermatitis, cancers and depending upon the types of exposure and types of devices/machines and duration of exposure. Lifestyle: lack of physical activities, poor nutrition and unhygienic-insanitary conditions, sleep deprivation and chronic fatigue, emotional distress, depression, anxiety stress, and relationship difficulties can exaggerate the diseases and disorders due to exposures. Lack of yoga, meditation and physical exertions/ exercises and nutrition and continuous use of gadgets and addiction and craving for using particular types of devices e.g. craving for using social media software applications on smartphones, and craving for playing games on various gadgets can also exaggerate the severity of the diseases and disorders due to exposure [7]. Therefore, to save ourselves, from the adverse effects of gadgets the suggested screen time for 30 to 60 minutes in 24 hours and better health can be attained by intermittently yogic exercises, breathing exercises, meditation and physical exercises and balanced diet.

For keeping the physical dimension of health in normal range the nutrition, physical exercises, breathing exercises, yogic exercises will be helpful, for keeping the mental dimension of health in normal range the meditation, pranayama and yoga will be helpful, for keeping the social dimension of health in normal range the community meditation, community pranayama and community yoga will be helpful especially when carried out in the groups, for keeping the emotional dimension of health in normal range the meditation, pranayama and yoga will be helpful when practised along with the family members and eating along with family members and community eating will also be helpful, for keeping the spiritual dimension of health in normal range the offering prayers to the God, Surya Namaskar and intermittent visits will be helpful, for keeping the vocational dimension of health in normal range a good job or business with awesome salary or income will be helpful. So, finally I can conclude that least contact period with the devices/gadgets/appliances, least screen time to see social media updates, good foods, good social interactions, interactions with family members, 1-2 hours rigorous exercises with sweating, nice job or business, healthy working conditions, awesome salary or income, prayers, visits to religious places, visits to the places full of natural resources like dense woods, fountains, waterfalls, rivers, dams, mountains, sea beaches etc. can keep us healthy $[8,9]$.

\section{References}

1. Robert I (2016) Electronics. The history of electronics. Encyclopaedia Britannica, United Kingdom.

2. https://www.allaboutcircuits.com/textbook/experiments/

3. https://www.wired.com/brandlab/2015/04/rise-machines-futurelots-robots-jobs-humans/

4. https://gadgets.ndtv.com/

5. Sigman A (2012) The impact of screen media on children: A Eurovision for parliament. Improving the Quality of Childhood in Europe 3: 88-121.

6. https://www.indiatoday.in/india/north/story/yamunagar-earphoneson-crushed-to-death-93175-2012-02-15

7. Binorkar SV (2014) Yoga-The non-pharmaceutical approach for lifestyle disorders. Yoga Phys Ther 4: 4.

8. Leena K, Tomi L, Arja R (2005) The intensity of mobile phone use and health-compromising behaviours-how is information and communication technology connected to health-related lifestyle in adolescence? J Adolesc 28(1): 35-47.

9. Muduli JR (2014) Addiction to technological gadgets and its impact on health and lifestyle: A study on college students. 